\title{
TGFBI is involved in the formation of polyploid cancer cells and the response to paclitaxel
}

\author{
Xiaobin Shang ${ }^{1 \#}$, Bibo Yuan ${ }^{2 \#}$, Jingjing $\mathrm{Li}^{2}$, Fangfang $\mathrm{Xi}^{2}$, Jingxin Mao ${ }^{2}$, Chen Zhang ${ }^{1}$, Hongjing Jiang ${ }^{1}$, \\ Guoyan Liu ${ }^{2}$
}

${ }^{1}$ Department of Minimally Invasive Esophageal Surgery, Key Laboratory of Cancer Prevention and Therapy, National Clinical Research Center for Cancer, Tianjin Medical University Cancer Institute and Hospital, Tianjin, China; ${ }^{2}$ Department of Gynecology and Obstetrics, Tianjin Medical University General Hospital, Tianjin, China

Contributions: (I) Conception and design: G Liu; (II) Administrative support: H Jiang; (III) Provision of study materials or patients: B Yuan, J Li; (IV) Collection and assembly of data: F Xi, J Mao, C Zhang; (V) Data analysis and interpretation: X Shang; (VI) Manuscript writing: All authors; (VII) Final approval of manuscript: All authors.

\#These authors contribute equally to this work.

Correspondence to: Guoyan Liu. Department of Gynecology and Obstetrics, Tianjin Medical University General Hospital, No. 154, Anshan Rd., Heping District, Tianjin 300052, China. Email: liuguoyan211@126.com; Hongjing Jiang. Department of Minimally Invasive Esophageal Surgery, Key Laboratory of Cancer Prevention and Therapy, National Clinical Research Center for Cancer, Tianjin Medical University Cancer Institute and Hospital, Tiyuanbei, Huanhuxi Rd., Hexi District, Tianjin 300060, China. Email: jianghj@vip.163.com.

Background: Most human solid tumors are aneuploid; at the same time, polyploid cancer cells are found to be resistant to radiotherapy and chemotherapy and have a poor prognosis. The transforming growth factor beta induction (TGFBI) protein plays important roles in the development of tumors, depending on the cancer of origin.

Methods: In this study, we established polyploid clones of breast cancer treated with nocodazole. The drug sensitivity was measured by MTT assay. Western blot analysis was used to detect the expression of TGFBI protein in polyploid clones. The effects of paclitaxel on apoptosis, cell cycle and DNA ploidy were analyzed by flow cytometry. TGFBI protein expression was performed in samples from patients with epithelial ovarian tumors by immunohistochemical staining.

Results: We found that compared with the MDA-MB-231 cell line, the expression of TGFBI in the HGF1806 cell line was relatively higher. In addition, compared with its parental cells, TGFBI showed relatively low expression in the polyploid breast cancer cell line T-MDA-MB-231. Compared with the empty vector, under paclitaxel treatment, the over-expression of TGFBI in MDA-MB-231 and T-MDA-MB-231 both showed a higher growth inhibition rate. After nocodazole treatment, the over-expression of TGFBI in MDF-MB-231 cells proved that the expression of tetraploid cells was lower compared to the control. The positive rate of TGFBI expression in ovarian cancer specimens before chemotherapy was 33.3\% (5/15), which was higher than the positive rate of $T G F B I$ expression in ovarian cancer specimens matched with relapsed specimens after treatment $(0 \%, 0 / 15)$.

Conclusions: TGFBI can increase the sensitivity of paclitaxel in polyploid cancer cells and participate in the formation of polyploidy in MDA-MB-231 induced by nocodazole. This newly recognized role of TGFBI provides further insight into the pathogenesis of polyploid cancer and identifies potential new therapeutic targets.

Keywords: Polyploid cancer; transforming growth factor beta-induced (TGFBI); drug sensitivity; nocodazole; paclitaxel

Submitted Jan 27, 2021. Accepted for publication Apr 22, 2021.

doi: 10.21037/atm-21-1698

View this article at: http://dx.doi.org/10.21037/atm-21-1698 


\section{Introduction}

Chemotherapy plays an important role in the treatment of cancer. Paclitaxel, a spindle poison, has been widely used as an effective chemotherapy agent for the treatment of solid tumors, such as ovarian and breast cancer. The adverse reactions of paclitaxel are mainly manifested in several aspects such as allergic reaction, cardiotoxicity, bone marrow suppression. Under the premise of controlling the administration time, the adverse reactions can be minimized. Despite initial chemosensitivity, the majority of cancer patients will eventually relapse and die due to resistance. The key factors affecting the effectiveness of chemotherapy antagonism and recurrence are including the burden of tumor cells, tumor growth rate, blood vessel supply, cell division method. High, medium and low doses of paclitaxel have no significant effect on the transcription and protein synthesis of smooth muscle cell protooncogenes c-fos and c-jun. The development of a cellbased pathway modulator screening system to screen drug candidates for paclitaxel-like cancer therapeutic activity is a valuable resource.

Our previous study found that different breast cancer cell lines react differently to the spindle poison, nocodazole, with some experiencing apoptosis and others forming polyploidy(tetra-/octopolyploidy) with the prolonged exposing time. It is well known that polyploid cancer cells are frequently observed in premalignant and malignant cells. Accumulating evidence indicates that polyploid cancer cells are resistant to radiotherapy and chemotherapy (1). The existence of polyploid cell subclones in human tumors and the subsequent formation of polyploid tumors are associated with poor prognosis (2).

In one study, a nocodazole-induced stable polyploidy breast cancer cell line T-MDA-MB-231 was established which showed acquired resistance to most chemotherapy drugs currently used in clinic, as compared with the parental breast cancer cell line, MDA-MB-231 (3). This indicated that the formation of polyploid cell induced by spindle poison may be associated with chemotherapy resistance.

$T G F B I$ protein is a transforming growth factor beta $(T G F-\beta)$ inducible secreted extracellular matrix (ECM) protein, which is also known as $\beta i g-\mathrm{H} 3$, and is derived from its cloning in the lung adenocarcinoma cell line A549 (4). Secreted protein acidic and rich in cysteine (SPARC) can reduce the deposition of TGFBI in mesothelial-derived $E C M$ without affecting its overall protein expression or secretion in ovarian cancer. TGFBI is believed to be related to tumor suppression and tumor promotion, depending on the origin of the cancer (5). Specifically, it has been demonstrated that TGFBI is under-expressed in breast, ovarian, and lung cancers $(6,7)$, and overexpressed in renal clear cell carcinoma, colorectal cancer, and pancreatic cancer (8-10). Moreover, the loss of TGFBI expression is linked to centrosome duplication and chromosomal instability $(C I N)$, both which are associated with carcinogenesis and drug-resistant phenotypes (11-13). TGFBI can preferentially interacts through a $B 3$ integrin receptor mediated mechanism to regulate the response of cells to paclitaxel-induced cell death. Here, we found for the first time that $T G F B I$ had a relatively higher expression in T-MDA-MB-231 than in MDA-MB-231, and that TGFBI was involved in paclitaxel resistance in polyploid cancer cell. Is TGFBI really related to the formation of polyploidy? What role does it play in the formation of polyploidy? Is it related to paclitaxel resistance? The answers to these questions are still unclear. In our study, we established polyploid monoclonal cells and then carried out exploratory research from the level of cytology and patient histology. We found TGFBI can increase the sensitivity of paclitaxel in polyploid cancer cells and participate in the formation of polyploidy in MDA-MB-231 induced by nocodazole. This newly recognized role of TGFBI provides further insight into the pathogenesis of polyploid cancer and identifies potential new therapeutic targets.

We present the following article in accordance with the MDAR reporting checklist (available at http://dx.doi. org/10.21037/atm-21-1698).

\section{Methods}

\section{Cell lines, reagents, cell culture, and plasmid transfection}

Human breast cancer cell lines, MDA-MB-231 and HCC-1806, were propagated in RPMI 1640 medium supplemented with $10 \%$ fetal bovine serum in a $5 \% \mathrm{CO}_{2}$ incubator at $37^{\circ} \mathrm{C}$. A plasmid-expressing TGFBI and a negative control were obtained from OriGene (Rockville, USA). The cells were seeded in a 6 -well plate at $2 \times 10^{5} \%$ well and allowed to attach for at least 16 hours. The vector expressing TGFBI and the empty vector $(4 \mu \mathrm{g})$ were transfected with Lipofectamine 2000 (Invitrogen, Grand Island, NY, USA) at a final concentration of $20 \mathrm{nM}$. The protein was collected 48 hours after transfection. 
Establishment and characterization of a polyploidy breast cancer cell line

MDA-MB-231 cells in the exponential phase were treated with nocodazole $(100 \mathrm{ng} / \mathrm{mL})$ for 24,48 , and 72 hours. Cells were collected, and the cycle distribution was measured by flow cytometry using an FACScan flow cytometer (BD Biosciences, Franklin Lakes, NJ, USA).Cells were dyed with Hoechst 33342, and flow cytometry was used to sort out the polyploidy cells. To generate polyploid clones, the T-MDA-MB-231 cell was sorted in the same fashion and was then subcloned by limiting dilution into T-MDA-MB-231 polyploidy clones.

\section{In vitro drug sensitivity assay}

Drug sensitivity was measured by MTT assay. Twentyfour hours after being transfected with TGFBI-expressing plasmid or empty vector, the cells were seeded into a 96well plate $\left(1 \times 10^{3}\right.$ cells/well $)$ and treated with paclitaxel titration at $37{ }^{\circ} \mathrm{C}$ in a $5 \% \mathrm{CO}_{2}$ humidified atmosphere for 72 hours. After incubation, $20 \mu \mathrm{L}$ of MTT (Sigma Chemical, St. Louis, MO) was added to each well, and the plate was incubated for 4 hours, and then $200 \mu \mathrm{L}$ of dimethyl sulfoxide (DMSO) was added to measure $546 \mathrm{~nm}$ in spectrophotometry. The cell survival score was then calculated. Cell viability was also calculated by normalizing the absorbance to that of the untreated control.

\section{Western blot analysis}

The primary antibody for $\beta$-actin was obtained from Santa Cruz Biotechnology (Santa Cruz, CA, USA). TGFBI antibody was purchased from Abcam (Cambridge, UK). Western blotting was performed by loading the whole cell lysate $(30 \mu \mathrm{g})$ of each sample on a $10 \%$ polyacrylamide gel for electrophoresis. The membrane was blocked in 5\% skimmed milk in $1 \mathrm{x}$ Tris buffered saline solution $(\mathrm{pH} 7.4)$ containing $0.05 \%$ Tween-20 and used at a concentration of $1: 1,000$ (for $T G F B I$ ) or $1: 2,000$ (for $\beta$-actin). The concentration of the secondary antibody was $1: 10,000$ to 1:20,000. Pierce Chemical (Rockford, IL, USA)'s Super Signal West Pico or SuperSignal Femto Chemiluminescent Substrate was used to visualize proteins.

\section{Annexin-V-FITC/PI apoptosis assay}

The cells were labeled with Annexin-V-FITC/PI and analyzed by flow cytometry to detect the effect of paclitaxel apoptosis on the degree of apoptosis of MDA-MB-231 or T-MDA-MB-231 cells. After transfection of TGFBI plasmid or empty vector, MDA-MB-231 or T-MDA-MB-231 cells were exposed to $3.125 \mu \mathrm{M}$ of paclitaxel for 24 or 48 hours, and floating cells and adherent cells were combined and collected. Annexin-V-FITC/PI was dyed according to the manufacturer's instructions (B\&D Systems) (R\&D Systems, Minneapolis, $\mathrm{MN}$ ).

\section{Cell cycle and DNA ploidy analysis by flow cytometry}

Forty-eight hours after transfection, the cells were collected, washed with phosphate buffered saline (PBS), and fixed in $4 \% 70 \%$ ethanol overnight $70 \%$ ethanol at $4{ }^{\circ} \mathrm{C}$ overnight. After fixation, the cells were washed twice with PBS and then resuspended in propidium iodide/RNase A solution $(5 \mu \mathrm{g} / \mathrm{mL}$ propidium iodide and $100 \mathrm{mg} / \mathrm{mL}$ RNase A). The cells were incubated with propidium iodide in the dark for 1 hour at room temperature. Using the FACScan flow cytometer (BD Biosciences), the light scattering characteristics and DNA content of the stained cells were analyzed by the flow cytometer. The main cell cycle subphases, G0/1, S, G2/M, and DNA, were identified by direct quantitative measurement of DNA by a flow cytometer based on the fluorescent dye PI staining of the DNA. The integrated fluorescence peak value of the G0/1 population of untreated MDA-MB-231 cells was considered to be DI $=1.0$, and the DNA ploidy of the treated cells is expressed as the ratio of the peak fluorescence intensity of these cells to the cell apoptosis of untreated G0/1 cells. The peak value of integrated fluorescence of $\mathrm{G} 0 / 1$ population of untreated MDA-MB-231 cells is being considered to DI $=1.0$ and DNA ploidy of treated cells is expressed as a ratio of a peak value of fluorescence intensity of these cell with respect to that of the untreated G0/1 cells.

\section{Immunobistochemical staining}

Immunohistochemical staining was performed on 15 pairs of patient samples with epithelial ovarian tumor, which were assembled in our department. All patients accepted 7-11 cycles (median 8 cycles) of combined chemotherapy (including paclitaxel). The progression-free survival of all patients was shorter than 6 months. The median overall survival was 38.4 (range, 9-65) months. Rabbit antihuman polyclonal antibody against TGFBI (1:500, Abcam), EnVision+ System-HRP rabbit (Dako, Carpinteria, CA, 
A
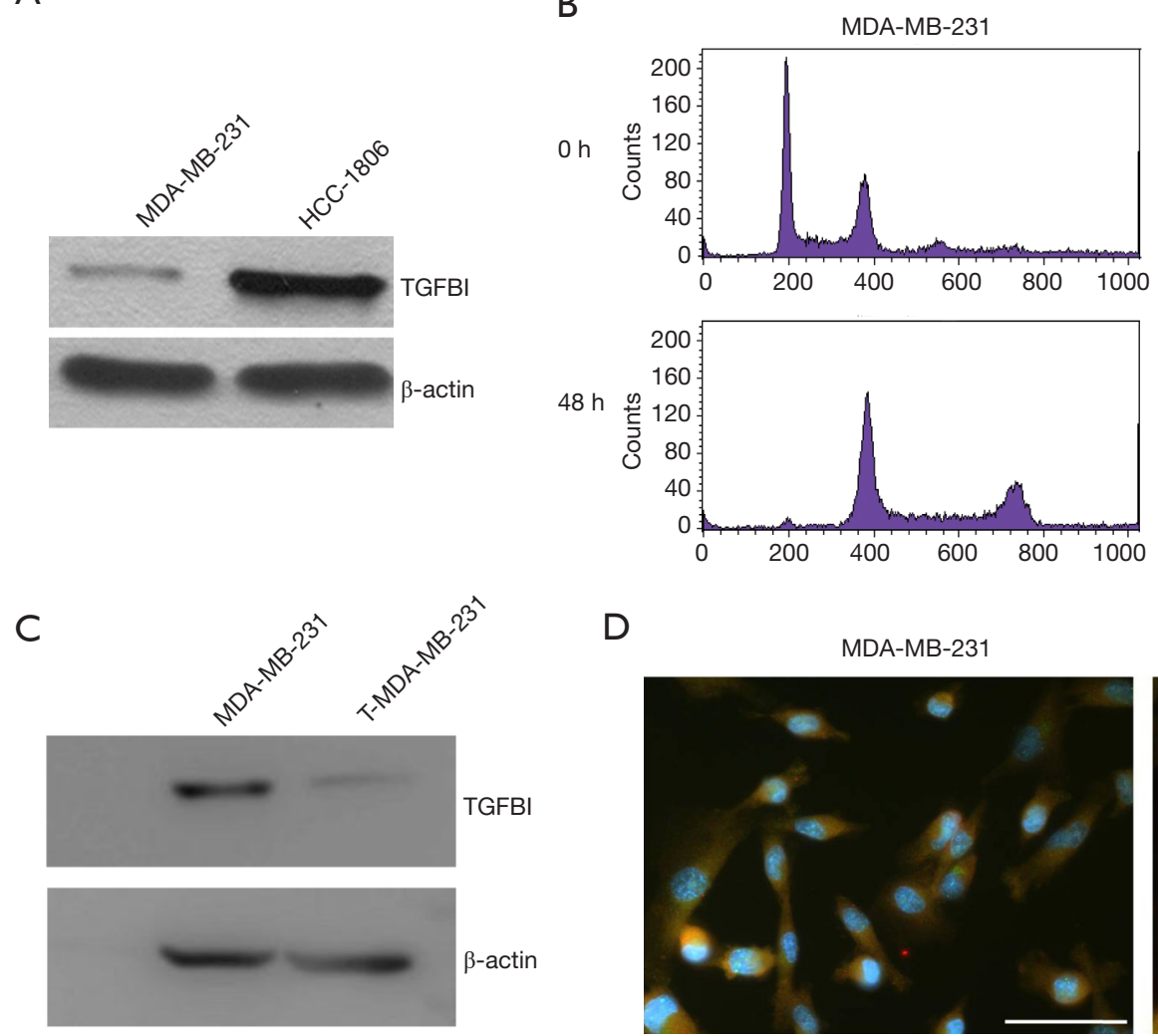

$\mathrm{Oh}$

B

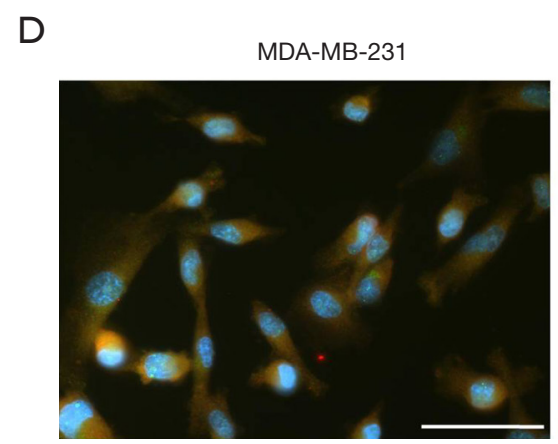

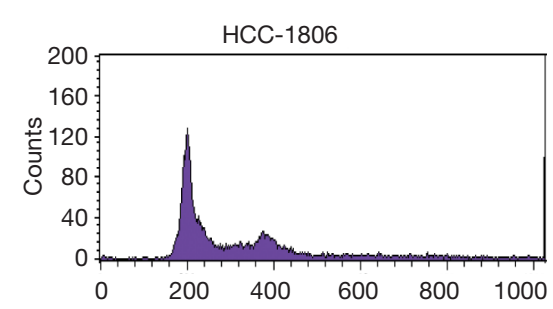

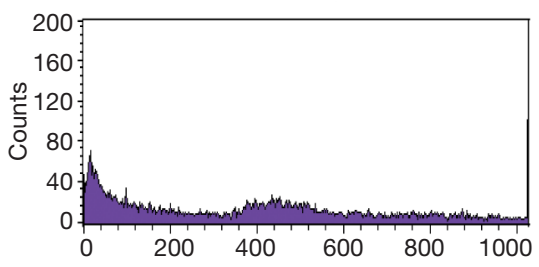

T-MDA-MB-231

Figure 1 TGFBI expression levels differed across HCC-1806, MDA-MB-231 andT-MDA-MB-231 cells. (A)Western blot results showing that TGFBI had a relatively lower expression in MDA-MB-231 cells than in HCC-1806 cells; (B) after 48 hours of nocodazole $(100 \mathrm{ng} / \mathrm{mL})$ treatment, the cell cycle distribution of MDA-MB-231 and HCC1806 was detected by flow cytometry; (C) the polyploidy breast cancer T-MDA-MB-231 cell line expressed relatively lower levels of TGFBI compared to its parent MDA-MB-231 cell line; (D) immunofluorescence images of MDA-MB-231 and T-MDA-MB-231 cells. Scale bar $=100 \mu \mathrm{m}$.

USA) was used. TGFBI-positive cells were defined as cells stained brown in the cytoplasm (primary) nucleus. Two independent, blinded observers evaluated the results of staining in all of the study samples.

The study was conducted in accordance with the Declaration of Helsinki (as revised in 2013). The Tianjin Medical University Ethics Committee gave approval for the experiments performed in this study, and informed consent was taken from all the patients.

\section{Statistical analysis}

Data are expressed as the mean \pm standard deviation (SD) of three independent experiments. Fisher's exact test was used to analyze the difference of TGFBI expression in clinical samples. All other analyses were performed using GraphPad
Prism version 6 software for Windows, and a P value $<0.05$ was considered statistically significant.

\section{Results}

TGFBI expression levels were different in HCC1806, MDA-MB-231, and T-MDA-MB-231 cells (Figure 1)

In our previous study, we found that HCC1806 and MDAMB-231 cells reacted quite differently to spindle poison, nocodazole (Figure 1B). HCC1806 underwent apoptosis, while MDA-MB-231 formed polyploidy with prolonged exposure time (Figure 1D). The level of TGFBI was examined in these two cell lines. The result showed that TGBFI was relatively more highly expressed inHCC1806 compared to MDA-MB-231 (Figure 1A). Furthermore, we compared the expression level of TGFBI in T-MDA- 
A

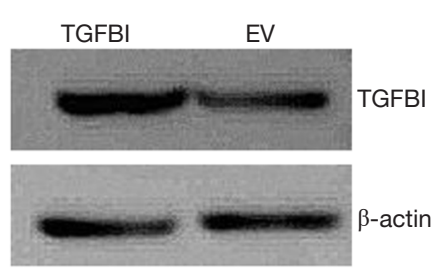

B

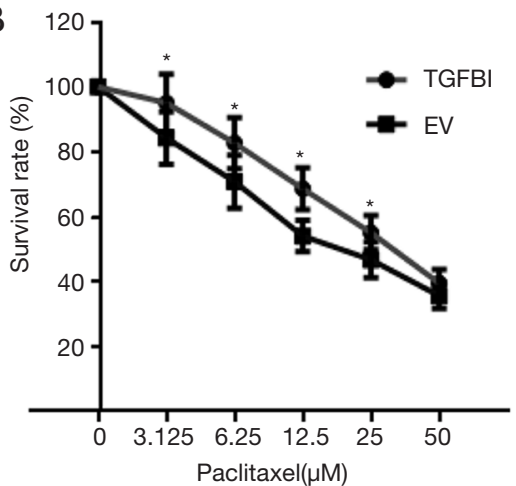

C

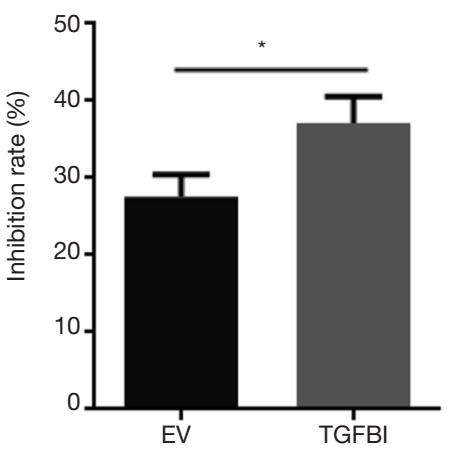

Figure 2 TGFBI sensitized polyploid cell line T-MDA-MB-231 to paclitaxel and nocodazole. T-MDA-MB-231 cells were transfected with $4 \mu \mathrm{g}$ of $T G F B I$-expressing vector or empty vector. After 24 hours, cells were reseeded for paclitaxel $\left({ }^{*}, \mathrm{P}<0.05\right)(\mathrm{B})$ sensitivity assay, $100 \mathrm{ng} / \mathrm{mL}$ of nocodazole $\left({ }^{*}, \mathrm{P}<0.05\right)(\mathrm{C})$ inhibition assay, or harvested for western blot analysis $(\mathrm{A})$. Generated curves and histograms from three independent experiments.

MB-231 to its parent MDA-MB-231. As expected, polyploidyT-MDA-MB-231 expressed a relatively lower TGFBI level (Figure 1C). Taken together, these results indicated that TGFBI may be associated with the formation of polyploidy.

\section{TGFBI sensitized polyploid cell line T-MDA-MB-231 to paclitaxel}

In our previous study, we found that T-MDA-MB-231 showed relative resistance to most commonly used chemotherapy drugs, including paclitaxel. As T-MDAMB-231 has a relatively lower expression of TGFBI than does MDA-MB-231, we over-expressed TGFBI using a TGFBI plasmid vector in T-MDA-MB-231 and evaluated its response to paclitaxel using an MTT assay. As expected, over-expression of TGFBI demonstrated a higher growth inhibition rate compared with the empty vector $(\mathrm{P}<0.05)$ (Figure 2). Thus, T-MDA-MB-231 cells transfected with $T G F B I$ were more sensitive to paclitaxel than the control.

\section{TGFBI sensitized MDA-MB-231 to paclitaxel and was involved in nocodazole-induced polyploidy formation of $M D A-M B-231$}

Compared with HCC-1806, which demonstrated apoptosis to nocodazole treatment, MDA-MB-231 expressed a relatively lower TGFBI level and formed polyploidy with longer exposure to nocodazole. The result of the MTT assay showed that over-expression of TGFBI could sensitize MDA-MB-231 to paclitaxel (Figure 3). To determine the difference in apoptosis proportion, 24 hours after transfection of TGFBI or empty vector, MDA-MB-231 cells were treated with $3.125 \mu \mathrm{M}$ of paclitaxel for 24 and 48 hours, and the level of apoptosis was measured using Annexin V/PI staining. Consistent with the result of MTT, flow cytometry results showed that over-expression of TGFBI induced a higher apoptosis rate than did the control $(\mathrm{P}<0.05,48$ hours after treatment with paclitaxel) in MDAMB-231 cells (Figure 3).

Because paclitaxel could induce $\mathrm{G}_{2} / \mathrm{M}$ arrest, we examined the cell cycle distribution of MDA-MB-231 cells over-expressing TGFBI after treatment with paclitaxel. MDA-MB-231cells transfected with $T G F B I$ vector displayed a greater percentage of cells in $G_{2} / M$ phase than did their counterparts transfected with empty vector 24 hours after treatment with $12.5 \mu \mathrm{M}$ of paclitaxel (Figure 4). This result suggested that this increased sensitivity effect of $T G F B I$ is at least partly due to a $\mathrm{G}_{2} / \mathrm{M}$ phase arrest.

Next, we examined the influence of TGFBI on polyploidy formation. As shown in Figure 5, 48 hours after $100 \mathrm{ng} / \mathrm{mL}$ of nocodazole treatment, over-expression of TGFBI demonstrated relatively lower tetrapolyploid cells $(12.08 \%)$ than did controls $(25.08 \%)$ in MDA-MB-231 cells $(\mathrm{P}<0.05)$.

\section{A low level of TGFBI was common in recurrent resistant ovarian cancer samples}

In order to determine whether loss of TGFBI correlates with polyploidy tumor and chemotherapy resistance, we retrospectively analyzed TGFBI protein expression and DNA polyploidy in a series of 15 pairs of advanced 

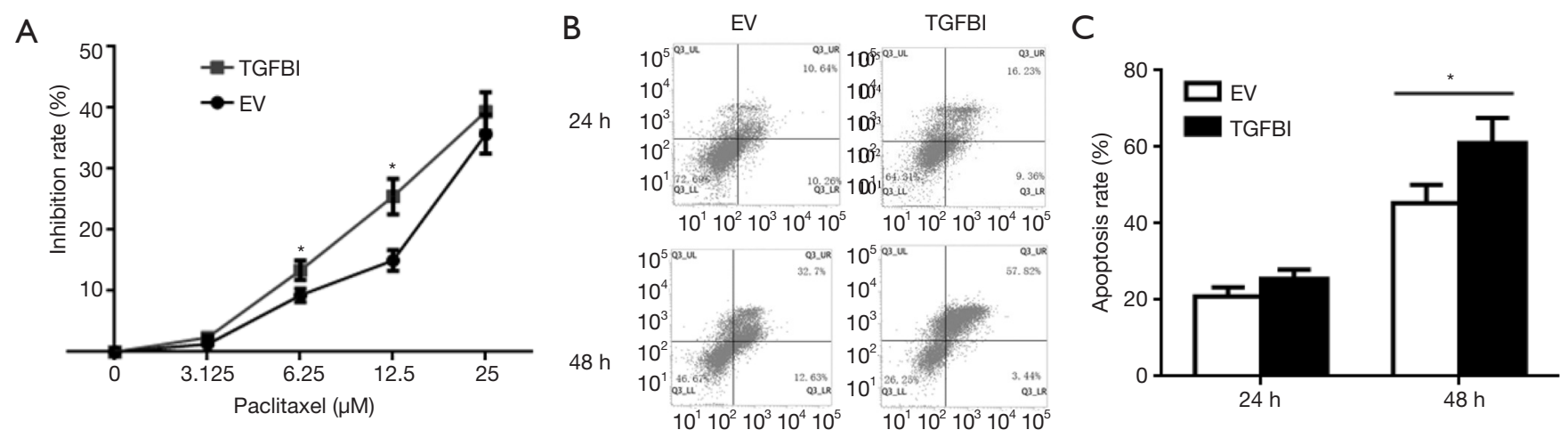

Figure 3 Transfection of TGFBI sensitized MDA-MB-231 to paclitaxel. (A) MDA-MB-231 cells were transfected with a vector expressing $4 \mu \mathrm{g}$ of TGFBI or an empty vector. After 24 hours, cells were reseeded for paclitaxel sensitivity assay (48 hours); (B) TGFBI significantly increased the apoptosis rate of MDA-MB-231 cells after treatment with $3.125 \mu \mathrm{M}$ of paclitaxel $\left({ }^{*}, \mathrm{P}<0.05\right)$. Annexin $\mathrm{V}$ assay results a shown. Three independent experiments were performed.

A

C
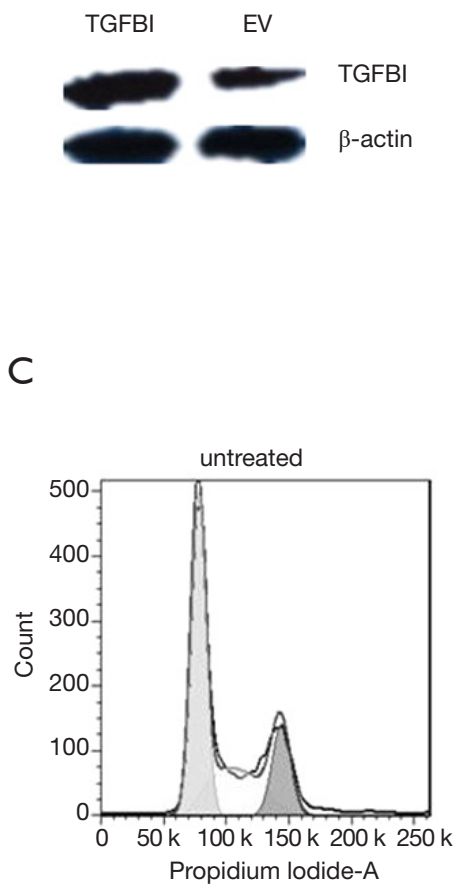

B

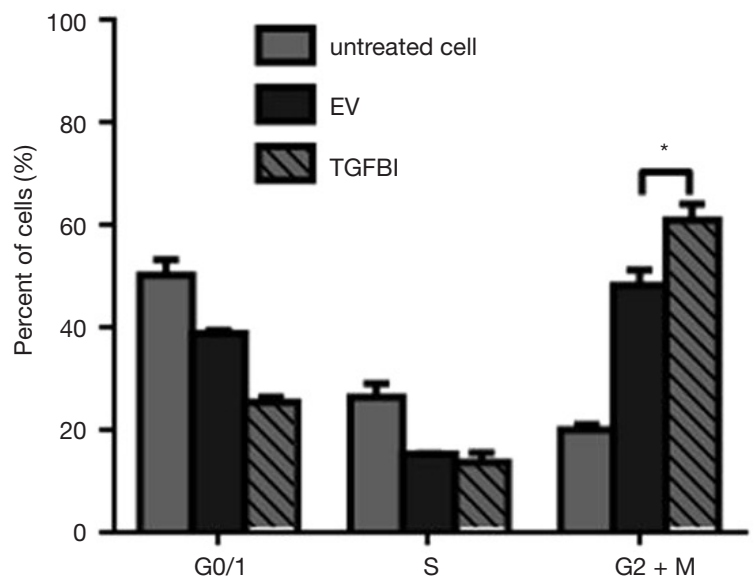

EV
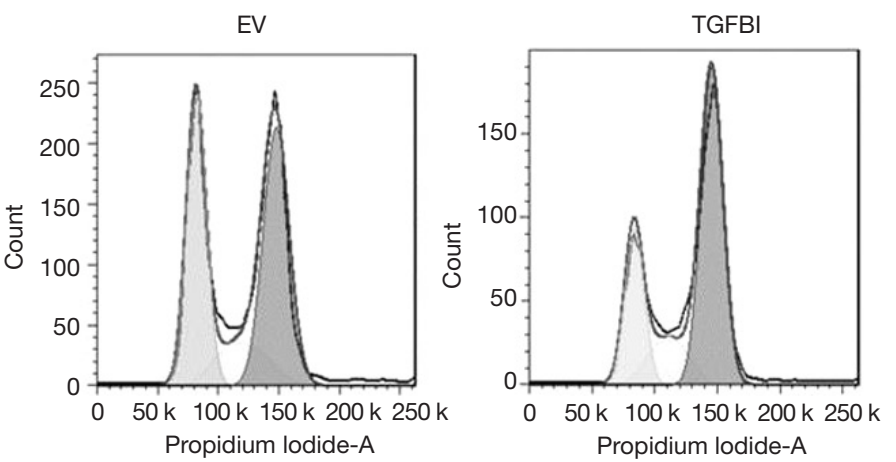

Figure 4 TGFBI enhanced the $\mathrm{G}^{2} / \mathrm{M}$ phase arrest induced by paclitaxel in MDA-MB-231 cells. (A) Western blot analysis of TGFBI expression in MDA-MB-231 cell 48hours after transfection of TGFBI vector or empty vector; (B) measurements of the cell cycle distribution of MDA-MB-231 cells after transfection with TGFBI or empty vector for 48 hours and treatment with $12.5 \mu M$ paclitaxel for 24 hours. *, $\mathrm{P}<0.05$; (C) representative images of flow cytometry for untreated cells and those treated with paclitaxel after transfection with TGFBI or empty vector. 
A

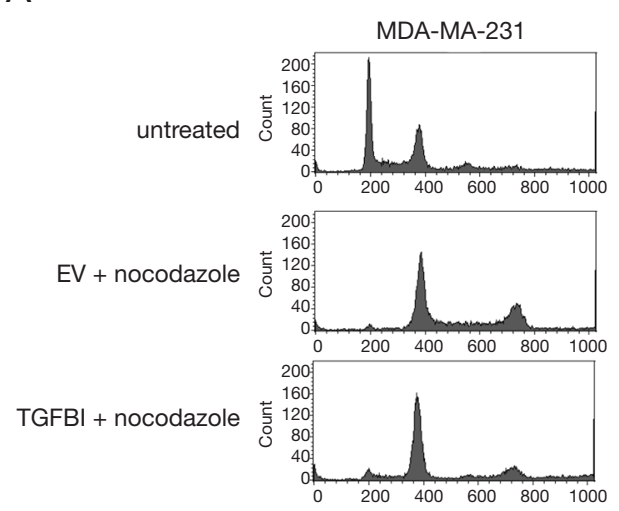

B

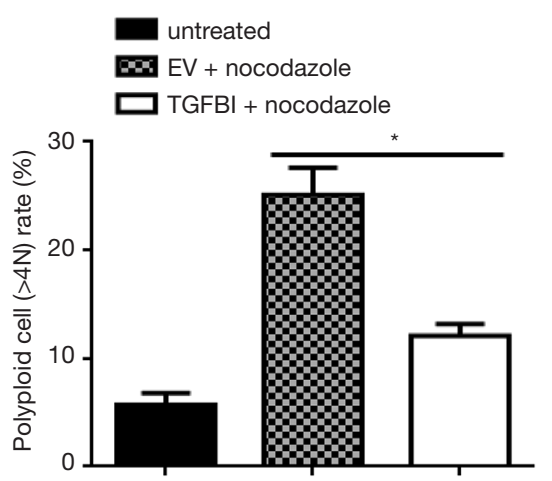

Figure 5 TGFBI decreased the polyploidy rate of MDA-MB-231 induced by nocodazole. Transfected MDA-MB-231 cells with a vector expressing $4 \mu \mathrm{g}$ of TGFBI or an empty vector. After 24 hours, cells were treated with $100 \mathrm{ng} / \mathrm{mL}$ of nocodazole for 24 or 48 hours. The DNA ploidy state was analyzed using flow cytometry. Representative images of flow cytometry (A) and results of the polyploidy cell rate (B) are shown $\left.{ }^{*}, \mathrm{P}<0.05\right)$. Three independent experiments were performed.

stage resistant ovarian cancer samples including initial (pre-chemotherapy) and matching recurrent (postchemotherapy) samples by immunohistochemistry and fluorescence in situ hybridization (FISH), respectively. The positive rate of TGFBI expression in samples before chemotherapy was $33.3 \%(5 / 15)$, which was higher than that of post-treatment and recurrent samples $(0 / 15$, Figure 6). Moreover, the amplified chromosome number significantly increased after recurrence compared to prechemotherapy (pending our published data).

\section{Discussion}

Most human solid tumors are aneuploid. The results of karyotype analysis of cancer cell lines show that the difference in the number of chromosomes from subdiploid to supertetraploid is very wide (2). In addition, direct evidence of progression from transient $4 \mathrm{~N}$ state (polyploidy) to aneuploidy has also been found in certain tumor types. Polyploid cancer cells are found to be resistant to radiotherapy and chemotherapy and to be related to poor prognosis.

Usually, mammalian somatic cells have complicated control mechanisms to prevent the occurrence of aneuploidy. Thus far, three different causes of tetraploidization in cancer have been proposed: cell fusion; cytokinesis failure; and endogenous duplication, including (endocycling and endomitosis forms).

The so-called spindle poisons, such as nocodazole, can induce polyploidy and aneuploidy through interfering with the formation of the spindle. Continuous exposure to nocodazole can cause a significant decrease in the depolymerization reaction of microtubules. In the presence of spindle defects, cells can exit mitosis and enter the next intermediate phase without chromatid separation, which is called mitotic slippage. Polyploidy is speculated to allow tumor cells to maintain a higher incidence of mutations, thereby increasing the possibility of adaptive changes.

TGFBI is secreted into the ECM as an attachment protein, and mainly plays a role in cell adhesion, migration, proliferation, apoptosis, and angiogenesis. Recently, it has been discovered that the TGFBI gene is often associated with the development of cancer. Depending on the tissue type and tumor microenvironment, the TGFBI protein may act in a variety of ways. The TGFBI gene is the downstream target of transforming growth factor $\beta(T G F-\beta)$. It exerts a tumor-suppressing effect in early tumorigenesis and acts as a tumor promoter in the later stage of tumor progression. This stage-specific dual-function role of TGFBI in cancer represents an emerging paradigm, and the underlying mechanism is not well understood. Recent findings indicate that TGFBI induces microtubule stabilization through integrin-mediated signaling pathways, thereby making ovarian cancer cells sensitive to paclitaxel, while the loss of TGFBI induces drug resistance and mitotic spindle abnormalities in ovarian cancer cells (13).

In our study, compared with parent MDA-MB-231 cells, polyploidy T-MDA-MB-231 cells expressed a 


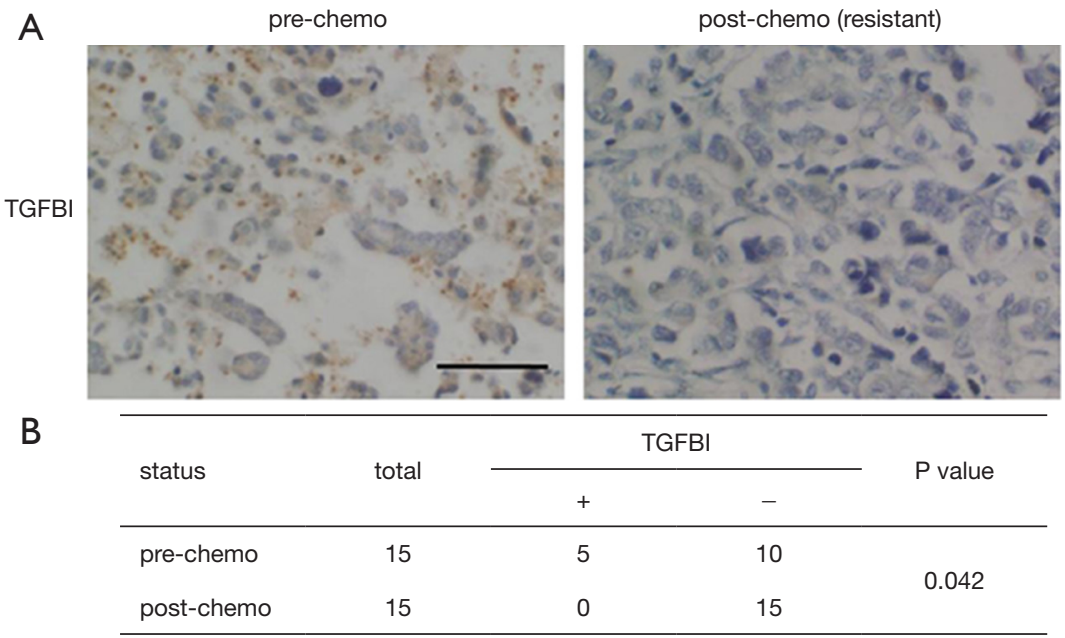

Figure 6 TGFBI expression was lower in recurrent resistant ovarian cancer samples compared with those of matching primary and prechemotherapy samples. (A) Representative images of immunohistochemical staining for TGFBI in pre- and post-chemotherapy samples are shown. Scale bar $=100 \mu \mathrm{m}$; (B) TGFBI expression information in primary pre-chemotherapy and recurrent post-chemotherapy ovarian cancer patients.

relatively lower level of TGFBI. Our MTT analysis showed that the cell inhibition rate of T-MDA-MB-231 cells was significantly increased after transfection of TGFBI compared to the controls, which suggested that the sensitivity of chemotherapy to paclitaxel was enhanced. To our knowledge, this is the first evidence that $T G F B I$ is associated with paclitaxel chemoresistance in polyploid cancer cells. Furthermore, we examined the formation of polyploidy after transfection of TGFBI induced by nocodazole. Results indicated that the rate of polyploidy cells decreased compared with controls in MDA-MB-231 cells when TGFBI was over-expressed. This result demonstrates that TGFBI, at the minimum, has a relationship with the formation of polyploidy induced by nocodazole. Recent literature has reported that loss of TGFBI expression is related to centrosome duplication and chromosomal instability, which implies a possible relationship between TGFBI and polyploidy. However, the potential mechanism remains to be elucidated.

Consistent with the results of in vitro experiments, TGFBI protein level in recurrent resistant ovarian cancer tissues was lower than that in matching primary tissues. In post-chemotherapy samples, the rate of polyploid state increased compared with that of the matching primary samples (pending our published data), which suggests that TGFBI is down-regulated in the process of formation of polyploidy, with polyploid tumors becoming concurrently resistant. Taken together, our findings provide evidence for the potential role of TGFBI in chemoresistance and formation of polyploidy.

\section{Conclusions}

TGFBI is related to paclitaxel response in polyploid cancer cells and to the formation of polyploid; it may thus have potential as a treatment tool for paclitaxel-resistant cancers.

\section{Acknowledgments}

Funding: This study was partially funded by two grants to GL from the Natural Science Fund of China (No. 81101673 and 81472761) and the Tianjin Science and Technology Committee Foundation (No. 14JCYBJC25300).

\section{Footnote}

Reporting Checklist: The authors have completed the MDAR reporting checklist. Available at http://dx.doi.org/10.21037/ atm-21-1698

Data Sharing Statement: Available at http://dx.doi. org/10.21037/atm-21-1698

Conflicts of Interest: All authors have completed the ICMJE uniform disclosure form (available at http://dx.doi. org/10.21037/atm-21-1698). The authors have no conflicts 
of interest to declare.

Ethical Statement: The authors are accountable for all aspects of the work in ensuring that questions related to the accuracy or integrity of any part of the work are appropriately investigated and resolved. The study was conducted in accordance with the Declaration of Helsinki (as revised in 2013). The Tianjin Medical University Ethics Committee gave approval for the experiments performed in this study, and informed consent was taken from all the patients.

Open Access Statement: This is an Open Access article distributed in accordance with the Creative Commons Attribution-NonCommercial-NoDerivs 4.0 International License (CC BY-NC-ND 4.0), which permits the noncommercial replication and distribution of the article with the strict proviso that no changes or edits are made and the original work is properly cited (including links to both the formal publication through the relevant DOI and the license). See: https://creativecommons.org/licenses/by-nc-nd/4.0/.

\section{References}

1. Etemadmoghadam D, Au-Yeung G, Wall M, et al. Resistance to CDK2 inhibitors is associated with selection of polyploid cells in CCNE1-amplified ovarian cancer. Clin Cancer Res 2013;19:5960-71.

2. Davoli T, de Lange $T$. The causes and consequences of polyploidy in normal development and cancer. Annu Rev Cell Dev Biol 2011;27:585-610.

3. Hao J, Yuan BB, Xu YF, et al. Sensitivity to chemotherapeutic drugs of polyploid tumor cells induced by a spindle poison nocodazole. Zhonghua Zhong Liu Za Zhi 2012;34:419-24.

4. Skonier J, Neubauer M, Madisen L, et al. cDNA cloning

Cite this article as: Shang $\mathrm{X}$, Yuan B, Li J, Xi F, Mao J, Zhang C, Jiang H, Liu G. TGFBI is involved in the formation of polyploid cancer cells and the response to paclitaxel. Ann Transl Med 2021;9(8):693. doi: 10.21037/atm-21-1698 and sequence analysis of beta ig-h3, a novel gene induced in a human adenocarcinoma cell line after treatment with transforming growth factor-beta. DNA Cell Biol 1992;11:511-22.

5. Thapa N, Lee BH, Kim IS. TGFBIp/betaig-h3 protein: a versatile matrix molecule induced by TGF-beta. Int $\mathrm{J}$ Biochem Cell Biol 2007;39:2183-94.

6. Calaf GM, Echiburú-Chau C, Zhao YL, et al. BigH3 protein expression as a marker for breast cancer. Int $\mathrm{J}$ Mol Med 2008;21:561-8.

7. Zhao Y, El-Gabry M, Hei TK. Loss of Betaig-h3 protein is frequent in primary lung carcinoma and related to tumorigenic phenotype in lung cancer cells. Mol Carcinog2006;45:84-92.

8. Yamanaka M, Kimura F, Kagata Y, et al. BIGH3 is overexpressed in clear cell renal cell carcinoma. Oncol Rep 2008;19:865-74.

9. Schneider D, Kleeff J, Berberat PO, et al. Induction and expression of betaig-h 3 in pancreatic cancer cells. Biochim Biophys Acta 2002;1588:1-6.

10. Buckhaults P, Rago C, St Croix B, et al. Secreted and cell surface genes expressed in benign and malignant colorectal tumors. Cancer Res 2001;61:6996-7001.

11. Zhang Y, Wen G, Shao G, et al. TGFBI deficiency predisposes mice to spontaneous tumor development. Cancer Res 2009;69:37-44.

12. Swanton C, Nicke B, Schuett M, et al. Chromosomal instability determines taxane response. Proc Natl Acad Sci U S A 2009;106:8671-6.

13. Ahmed AA, Mills AD, Ibrahim AE, et al. The extracellular matrix protein TGFBI induces microtubule stabilization and sensitizes ovarian cancers to paclitaxel. Cancer Cell 2007;12:514-27.

(English Language Editor: J. Gray) 